\title{
Oxygen Uptake Estimation in Humans during Exercise Using a Hammerstein Model
}

\author{
Steven W. Su ${ }^{\dagger *}$, Lu Wang ${ }^{\dagger}$, Branko G. Celler ${ }^{\dagger}$, and Andrey V. Savkin ${ }^{\dagger}$
}

\begin{abstract}
This paper aims to establish a block-structured model to predict oxygen uptake in humans during moderate treadmill exercises. To model the steady state relationship between oxygen uptake (oxygen consumption) and walking speed, six healthy male subjects walked on a motor driven treadmill with constant speed from 2 to 7 kilometer/hour. The averaged oxygen uptake at steady state $\left(\mathrm{VO}_{2}\right)$ was measured by a mixing chamber based gas analysis and ventilation measurement system (AEI Moxus Metabolic Cart). Based on these reliable date, a nonlinear steady state relationship was successfully established using Support Vector Regression methods. In order to capture the dynamics of oxygen uptake, the treadmill velocity was modulated using a Pseudo Random Binary Signal (PRBS) input. Breath by breath analysis of all subjects was performed. An ARX model was developed to accurately reproduce the measured oxygen uptake dynamics within the aerobic range. Finally, a Hammerstein model was developed, which may be useful for implementing a control system for the regulation of oxygen uptake during treadmill exercises.
\end{abstract}

Keywords: Oxygen uptake, Support Vector Regression, Hammerstein model, identification, treadmill exercise, PRBS

\footnotetext{
${ }^{\dagger}$ Human Performance Group, Biomedical Systems Lab, School of Electrical Engineering \& Telecommunications University of New South Wales,UNSW Sydney N.S.W. 2052 Australia.

${ }^{*}$ The corresponding author is currently with Faculty of Engineering, University of Technology, Sydney, Australia. Email: Steven.Su@uts.edu.au. Tel: +61 (2) 95147603.
}

This research was supported by the Australian Research Council (Grant DP0452186). 


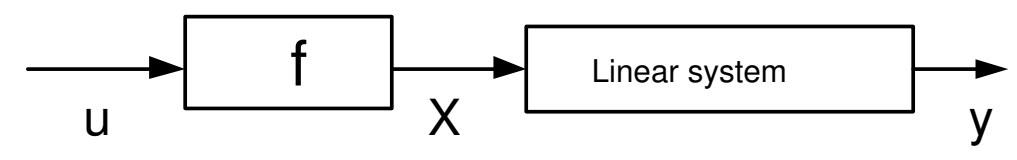

Fig. 1. A Hammerstein system.

\section{INTRODUCTION}

Oxygen uptake is an important physiological parameter for the determination of functional health status and clinical assessments in normal and pathological conditions. The main goal of this paper is to establish practical models to dynamically estimate oxygen uptake for walking exercisers. These models are potentially applicable for the regulation of oxygen uptake during treadmill exercise.

Papers about oxygen uptake modeling for moderate exercise can be divided into two categories: oxygen uptake estimation for the steady state condition (oxygen consumption $\mathrm{VO}_{2}$ ) and dynamic response characterization during onset and offset of exercise. For the prediction of steady state oxygen uptake, Franklin [20] proposed a linear static model to approximately estimate oxygen consumption in a given range of walking speed. Papers [14] [41] [11] provided simple static nonlinear (polynomial) models.

The oxygen uptake kinetic description at the onset and offset of leg work was first reported by Hill and Lupton [24] in 1923. After that, many papers attempt to capture both the exact time course of these kinetic adjustments and the physiological events governing the rate of adjustment. Most of them [34] [47] approximate the process by using first order linear models, which were often obtained by using step change responses. Hoffmann et al [25] [16] applied PRBS and sinewave signals to bicycle exercises. By using spectrum analysis, they proved the dynamic linearity of oxygen uptake with work load at low frequency.

In this paper, the steady state and dynamics of oxygen uptake during treadmill walking will be captured by using only one Hammerstein model. A novel model identification method for the Hammerstein model will also be presented.

The Hammerstein model can be described as a static nonlinear block followed by a dynamic linear system (see Figure 1). Hammerstein models may account for nonlinear effects encountered in not only industrial processes [17] [37], but also physiological processes [1] [26]. For example, 
electrically stimulated muscle [6] and lung tissue strip mechanics [30] can be effectively modeled using Hammerstein models.

The reason for using a Hammerstein model to predict oxygen uptake from treadmill speed is based on the following facts. The steady state relationship from treadmill speed to work load is not necessarily linear. The dynamic linearity from work load to oxygen uptake was proved by [25]. Therefore, it is reasonable to describe the relationship from treadmill speed to oxygen uptake by a nonlinear static element cascaded by a linear dynamic element.

The modeling of Hammerstein model is a very active research topic [29] [5] [21] [31] [3] [2] [4] [9] [7] [12] [45] [33] [38]. Recently, Goethals et al [21] presented a novel overparameterization (two-stage procedures [19]) identification approach for Hammerstein systems. The most distinguishing part of that approach is the utility of a powerful machine learning method, Least Square Support Vector Machine (LS-SVM) [39]. This novel machine learning method sets that paper apart from existing papers.

Support Vector Machine based regression [15] (Support Vector Regression (SVR)) is a new technique, which has been successfully applied to nonlinear function estimation. Vapnik et.al established and developed the foundation of SVM [42] [44]. SVR is very efficient in terms of speed and complexity, and successfully solves the so called over-fitting problem [22] by introducing regularization techniques.

This paper applies SVM approaches combining with stochastic method [5] to identify physiological processes. Our approach differs in at least two important aspects from that of paper [21]:

The stochastic method [2] is employed in preference to the over-parameterization method [5]. As discussed in [5] and [32], the error of the identification of Hammerstein model is not only from linear and static nonlinear part themselves but also from the coupling between them. Decoupling of the linear system identification from that of the static nonlinearity, through use of a stochastic input, is well known and follows from Bussgang's result [8]. Korenberg [28] carried out first the decoupling of the linear element identification for a more general block oriented model, a so-called LNL (dynamic linear/static nonlinear/dynamic linear) model. More over, Hunter and Korenberg [27] detail an iterative method for estimating the elements of a Hammerstein model that similarly exploits the decoupling of the linear and nonlinear identification steps. In this study, the pseudo-random binary sequences (PRBS) are applied to decouple the identification of 
the two parts as suggested in [5].

Another main difference of this approach with [21] is the usage of $\epsilon$-insensitivity SVM [10] [36] instead of LS-SVM [18] [39] [40]. Both LS-SVM and $\epsilon$-insensitivity SVM have the merits of SVM approaches. However, the loss function used by $\epsilon$-insensitivity SVM, only penalizes errors greater than a threshold $\epsilon$. This leads to a sparse representation of the decision rule giving significant algorithmic and representation advantages [10]. On the other hand, the ridge regression $(\epsilon=0)$ used by LS-SVM typically causes the loss of sparseness representation.

It should be emphasized that the proposed Hammerstein model identification approach is especially suitable for the identification of oxygen uptake model. For steady state oxygen uptake measurement, the most suitable way is to employ a mixing chamber to interrupt gas flow and thus prevent streaming of gases and uneven gas concentrations. However, gas samples collected from a mixing chamber are averaged gas fractions over time. The sensitivity to changes in oxygen uptake is therefore reduced [46]. In order to capture dynamic characteristics, breath by breath analysis should be applied for dynamic modeling experiments. However, this may lead to the degrading of measurement accuracy in steady state test. Due to the total decoupling of static part identification (based on steady state experiment data) and dynamic part identification (based on dynamic experiment data), it is appropriate to apply different measurement approaches for steady state tests and dynamic experiments respectively. As a consequence, better estimation results are attainable.

The paper is organized as follows. The details of SVM based Hammerstein model identification approach is given in Section 2. Section 3 presents the application of the proposed approach for the modeling of oxygen uptake during treadmill exercises.

\section{PROPOSED SVM BASED HAMMERSTEIN MODEL IDENTIFICATION APPROACH}

In [5], Bai showed that the identification of linear part of a Hammerstein model can be decoupled from nonlinear part with the help of the PRBS input. The reason is any static nonlinearity can be exactly characterized by a linear function under PRBS input which has a binary nature. In this study, the PRBS input is also employed. Thus, the identification of Hammerstein model can be obtained by the identification of static nonlinearity and linear dynamic separately.

As suggested in [5], the steady state gain of the linear dynamic model is constrained to be 
unity. The steady state characteristic of the Hammerstein system is considered by the static nonlinearity. It should be mentioned that for the identification of the static nonlinearity part, steady state experiments should be carried out because the PRBS inputs may not adequately excite the nonlinearity in our range of interest. Now, we introduce the so called -insensitivity SVR based static nonlinearity modeling first [43].

Let $\left\{u_{i}, y_{i}\right\}_{i=1}^{N} \subseteq \mathcal{R}^{d} \times \mathcal{R}$ be the inputs and outputs of the data of a Hammerstein system measured in steady state. The goal of the support vector regression is to find a function $f(u)$ which has the following form

$$
f(u)=w \cdot \phi(u)+b,
$$

where $\phi(u)$ represents the high-dimensional feature spaces which are nonlinearly transformed from $u$. The coefficients $w$ and $b$ are estimated by minimizing the regularized risk function:

$$
\frac{1}{2}\|w\|^{2}+C \frac{1}{N} \sum_{i=1}^{N} L_{\epsilon}\left(y_{i}, f\left(u_{i}\right)\right)
$$

The first term is called the regularized term. The second term is the empirical error measured by $\epsilon$-insensitivity loss function which is defined as:

$$
L_{\epsilon}\left(y_{i}, f\left(u_{i}\right)\right)=\left\{\begin{array}{cl}
\left|y_{i}-f\left(u_{i}\right)\right|-\epsilon, & \left|y_{i}-f\left(u_{i}\right)\right|>\epsilon \\
0, & \left|y_{i}-f\left(u_{i}\right)\right| \leq \epsilon
\end{array}\right.
$$

This defines a $\epsilon$ tube. The radius $\epsilon$ of the tube and the regularization constant $C$ are both determined by user.

By solving this constrained optimization problem, we have

$$
f(u)=\sum_{i=1}^{N} \beta_{i} \phi\left(u_{i}\right) \cdot \phi(u)+b .
$$

As mentioned before, by the use of kernels, all necessary computations can be performed directly in input space, without having to compute the map $\phi(u)$ explicitly. After introducing kernel function $k\left(u_{i}, u_{j}\right)$, the above equation can be rewritten as follows.

$$
f(u)=\sum_{i=1}^{N} \beta_{i} k\left(u_{i}, u\right)+b .
$$

Where the coefficients $\beta_{i}$ corresponding to each $\left(u_{i}, y_{i}\right)$, and only the so-called support vectors can have nonzero coefficients. 


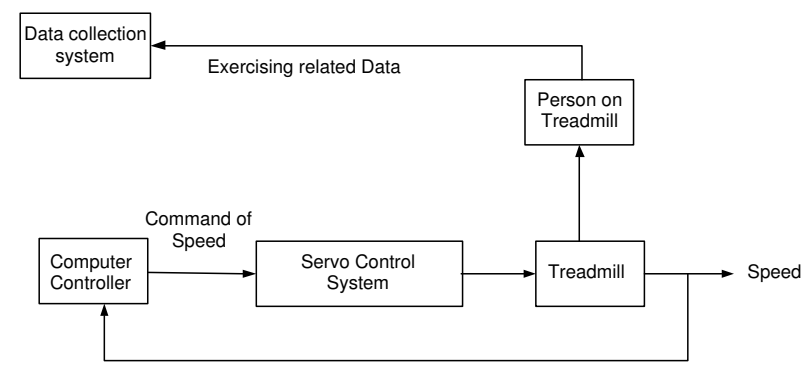

Fig. 2. Block diagram for experimental settings

For linear support regression, the kernel function is thus the inner product in the input space:

$$
f(u)=\sum_{i=1}^{N} \beta_{i}<u_{i}, u>+b .
$$

For nonlinear SVR, there are a number of kernel functions which have been found to provide good generalization capabilities, such as polynomials, Radial basis function (RBF), sigmod. Here we give the RBF and polynomials kernel functions as follows:

RBF kernel: $k\left(u, u^{\prime}\right)=\exp \left(-\frac{\left\|u-u^{\prime}\right\|^{2}}{2 \sigma^{2}}\right)$,

Polynomial kernel: $k\left(u, u^{\prime}\right)=\left(\left(u \cdot u^{\prime}\right)+b\right)^{d}$.

Detailed discussion about SVR, such as the selection of radius $\epsilon$ of the tube, kernel function, and the regularization constant $C$, can be found in [35] [43].

When PRBS input is employed for the identification of Hammerstein systems, as shown in equation (2.3) of [5], the identification of a Hammerstein model can be simplified as a linear identification problem. Any linear identification approach can be applied. Here, the parametric approach as suggested in [5] is adopted.

\section{Modeling OF OXYGen UPTAKe By USing A Hammerstein MOdel}

In this section, a Hammerstein model will be set up to estimate oxygen uptake from walking speed by using the approach described above.

\section{A. Experimental equipments}

The computer controlled treadmill and its related data collection and processing system are shown in figures 2 and 3. 


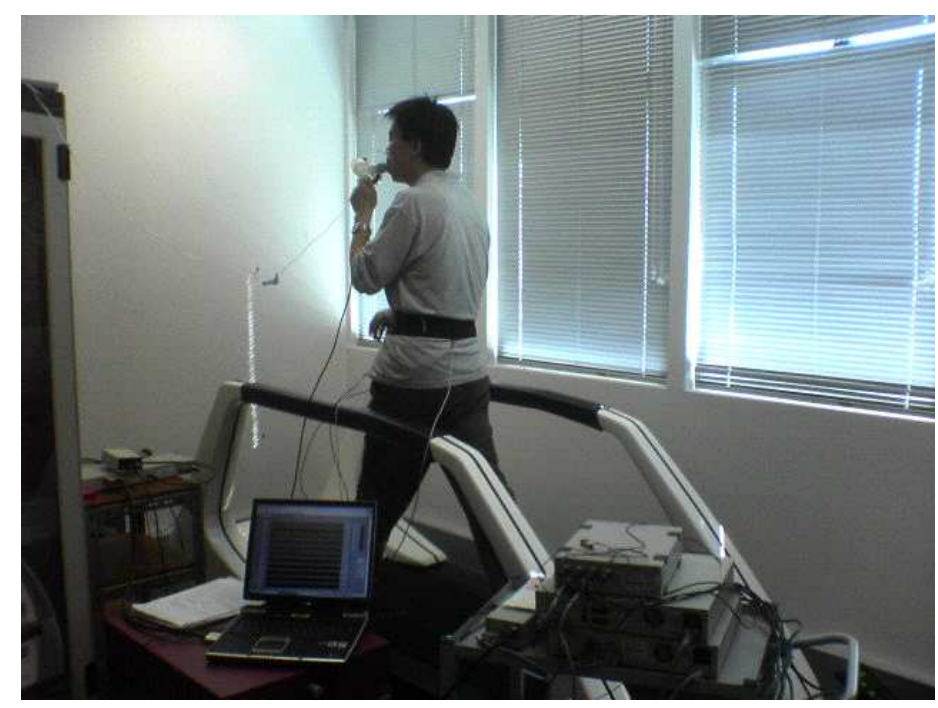

Fig. 3. The computer controlled treadmill system

The treadmill used in the system is the Powerjog "G" Series fully motorized medical grade treadmill manufactured by Sport Engineering Limited, England. Control of the treadmill can be achieved through an RS232 serial port. The treadmill can receive commands from the computer controller via this link, and obeys such commands without supervision. In order to implement PRBS type signal on treadmill, a computer based control system is implemented which can control the speed of the treadmill with a response time of less than 3 seconds. This is approximately twenty times faster than the increase in oxygen consumption that follows an increase in workload. During experiments, all signals are synchronized with the PRBS signal. The synchronization of signals was monitored using body worn triaxial accelerometers.

The measurement of oxygen uptake (either averaged or breath by breath) is implemented by using the AEI Moxus Metabolic Cart.

\section{B. Nonlinear component modeling by using Support Vector Regression}

In order to identify the nonlinear relationship, steady state experiments were performed. Six young healthy male subjects volunteered to participate in the study. Their physical characteristics are presented in Table I.

All experiments were conducted in the afternoon, and the subjects were permitted to have a light meal one hour before measurements were recorded. Initially, the subjects were asked to 


\begin{tabular}{cccc}
\hline & Mean & SD & Range \\
\hline Age $(\mathrm{yr})$ & 31.61 & 5.78 & $23-37$ \\
\hline Height $(\mathrm{cm})$ & 176.41 & 5.48 & $169-184$ \\
\hline Body mass $(\mathrm{kg})$ & 74.31 & 9.35 & $60-85$ \\
\hline
\end{tabular}

TABLE I

SUBJECT CHARACTERISTICS $(\mathrm{N}=6)$

walk for about 10 minutes on the treadmill to familiarize themselves with the experiment. The subjects were then requested to walk at six levels of different speeds $(2,3,4,5,6$ and $7 \mathrm{~km} / \mathrm{h})$. Each level took a total period of 5 minutes, and was followed by a 10-minute resting period. The oxygen uptake was recorded and averaged every two minutes by using a mixing chamber based gas analysis and ventilation measurement system (AEI Moxus Metabolic Cart). Finally, in order to identify linear dynamic part of the Hammerstein system, subjects were also requested to walk on the treadmill under a PRBS input. Throughout the experiments, the breath by breath tidal volume and the concentration of oxygen were recorded to calculate breath by breath oxygen uptake. The outputs of triaxial accelerometers were also recorded.

In this study, both traditional linear regression (see Figure 4) and the $\epsilon$-insensitivity SVR regression method are applied to modeling the nonlinear part. The regression error (Root Mean Square error) of SVR (1.66) is found to be much smaller than the linear regression error (2.17). The SVR regression results are summarized in Table ?? and Figure 5. In figures 4 and 5, the continuous curve stands for the estimated input output steady state relationship. The dotted lines indicate the $\epsilon$-insensitivity tube. The plus markers are the points of input and output data. The circled plus markers are the support points.

\section{Modeling for linear dynamic part}

Although powerful nonlinear regression method (SVR) is applied for the estimation of static nonlinear function, a steady state modeling error is inevitable (see Table ?? and Figure 5). Some commonly used Hammerstein model identification methods need to use the obtained static function when estimating the linear dynamic part. This will introduce coupling errors for the identification of the linear dynamic model as shown in [5] [21]. In order to avoid this coupling 


\begin{tabular}{ccccccc}
\hline Subjects & 1 & 2 & 3 & 4 & 5 & 6 \\
\hline $\begin{array}{c}\text { Time constant } \\
\text { (Seconds) }\end{array}$ & 45.4 & 61.8 & 69.3 & 63.3 & 65.8 & 64.1 \\
\hline $\begin{array}{c}\text { Noise variance } \\
\text { of models }\end{array}$ & 0.0013 & 0.0018 & 0.002 & 0.0017 & 0.0015 & 0.002 \\
\hline
\end{tabular}

TABLE II

TIME CONSTANT AND NOISE VARIANCE OF MODELS.

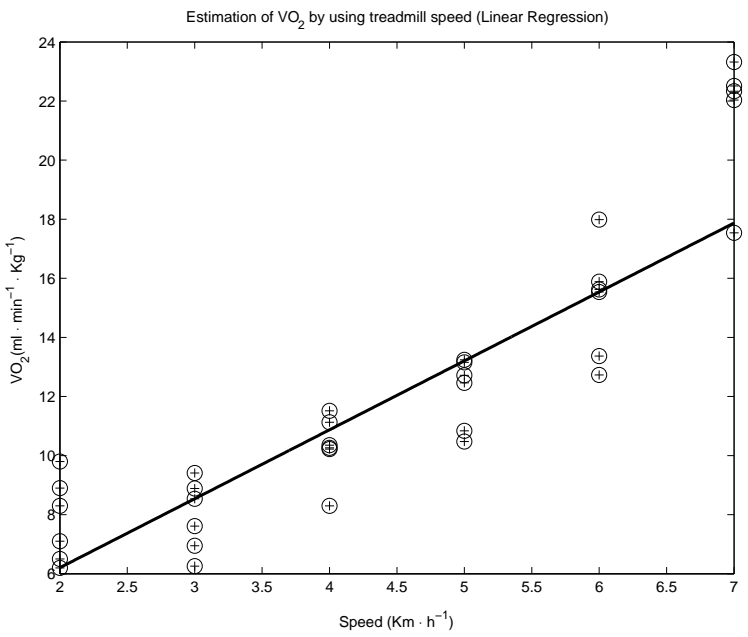

Fig. 4. The steady state relationship estimated by using linear regression

error, a well designed PRBS input (see Figure 6) is implemented in the automated treadmill system. A PRBS has two levels $(a+$ and $a-)$ and switches from one level to the other at constant time intervals $\Delta$. It is periodic with period $T=\Delta N$, where $N=2 n-1$ and $n$ is an integer. In order to avoid nonlinear behavior, the difference of the two levels of PRBS should be as close as possible. However, it is also required that the output responses under these two levels of inputs should be noticeably different (good signal to noise ratio) to ensure a reasonable parameter estimation results. For the selection of $\Delta$ and $N$, we need to compromise with the complexity of the selected model, response time of the system, noise level, and the total experimental time which the subjects can tolerate. In this study, we select $a+=6 \mathrm{~km} / \mathrm{h}$, $a-=4 \mathrm{~km} / \mathrm{h}, N=7$ and $\Delta=150$ seconds after several pre-experiments and detailed analysis 


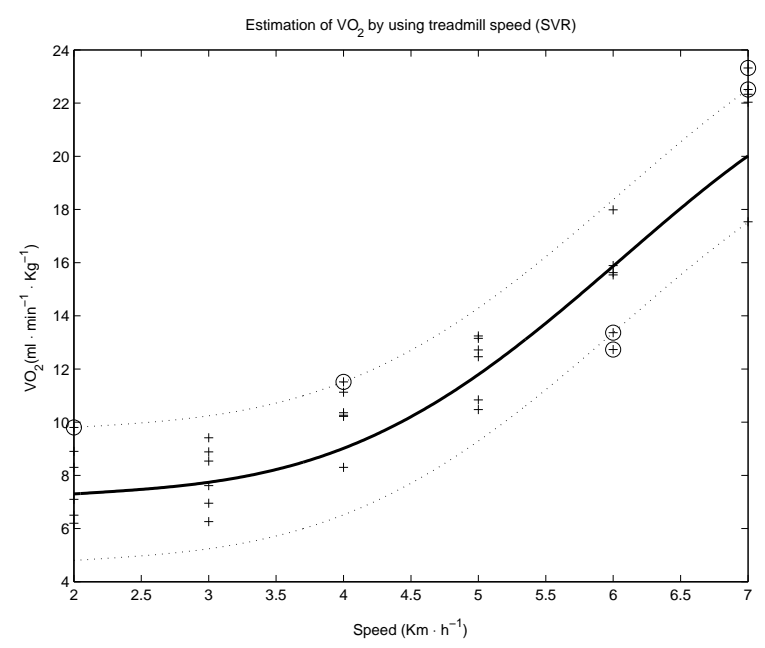

Fig. 5. The steady state relationship captured by using SVR

of the modeling output.

The oxygen uptake of all six subjects under the PRBS input is shown in Figure 6. The averaged oxygen uptake of six experiments is shown in Figure 7. Papers [24] [13] [23] often select first order exponential, with no time delays to describe the dynamics of oxygen uptake. In this study, we also adopt first order exponential to describe the dynamics. However, the time delays will be explored in this study because time delay is a crucial parameter for control system design. Neglecting of time delays often introduces instability in the controlled closed loop. In this study, the triaxial accelerometers are employed to synchronize body movements with respiratory related signals, which ensures reliable estimation of time delays. Based on the averaged data of PRBS input experiments, the time delay is determined by using Matlab Identification Toolbox. Two popular model selections criteria, Minimum Description Length (MDL) and Akaike Information Criterion (AIC), both give the following linear model:

$$
y(k)=0.9308 y(k-1)+0.0692 u(k-1)+e(k),
$$

with sampling period $T_{s}=5$ seconds. The noise variance of model (7) is $3.5 \times 10^{-4}$. By transferring model (7) to its corresponding continuous form (8)

$$
Y(s)=\frac{1}{69.735 s+1} U(s),
$$

we can see that the dynamic part is a first order stable system (with time constant $T=69.7$ 


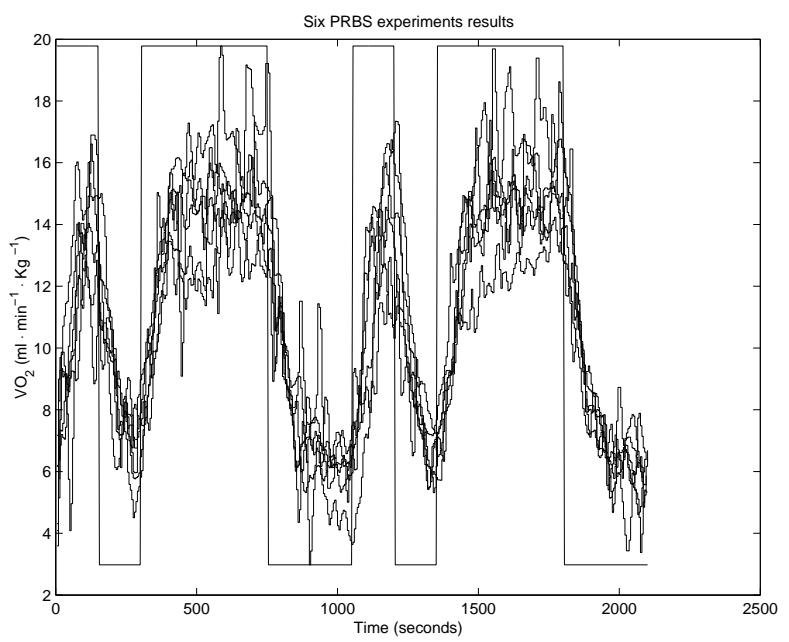

Fig. 6. The oxygen uptake of all six subjects under a two periods of 7 bits PRBS input

seconds) without time delay. This supports the belief [24] [13] [23] that the exponential rise in oxygen uptake directly reflectes the rate of rise and drop in leg muscle oxygen consumption $\left(\dot{Q} O_{2}\right)$ at the onset and offset of exercises. As the steady state characteristic of the overall Hammerstein system is considered by the static nonlinearity, the steady state gain of model (8) is constrained to be unity. The model fitting result is shown in Figure 7. Time constants and noise variances of models for each subject are summarized in Table II.

TABLE III. Time constant and noise variance of models.

\section{CONCLUSiON}

This paper uses a Hammerstein model identification method to estimate oxygen uptake in humans engaged in treadmill walking exercises. The identification of dynamic linear component is decoupled by using a PRBS signal. In order to obtain good modeling results, breath by breath 


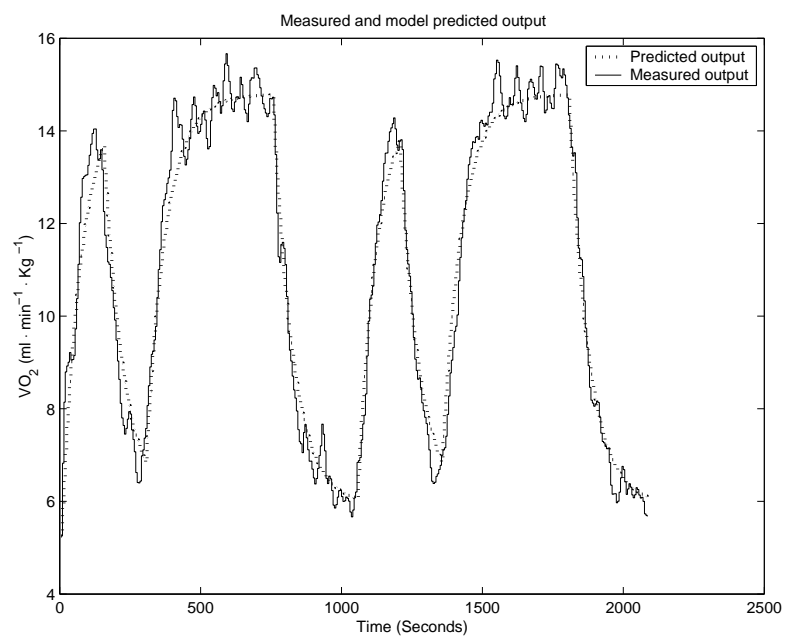

Fig. 7. Model estimation results

analysis is performed for PRBS input dynamic experiments. It is found that the time constants of identified first order ARX model for increasing and decreasing workload are identical within the aerobic range. This is an advantage for the design of controllers of oxygen uptake regulation for moderate treadmill exercises. By using the outputs of triaxial accelerometers to synchronize body movements with oxygen uptake, model time delays are investigated. Our results support the view that time delay of the oxygen uptake dynamics can be ignored. Oxygen uptake of steady state experiments are calculated by using mixing chamber based respiratory measurement system. A RBF kernel SVM model is achieved based on reliable steady state data, which is much better than traditional linear regression results. We believe that the proposed model may be useful in the modeling and regulation of oxygen uptake during treadmill exercises.

\section{A. Acknowledgments}

The authors gratefully acknowledge the financial support of the Australian Research Council (Grant DP0452186). 

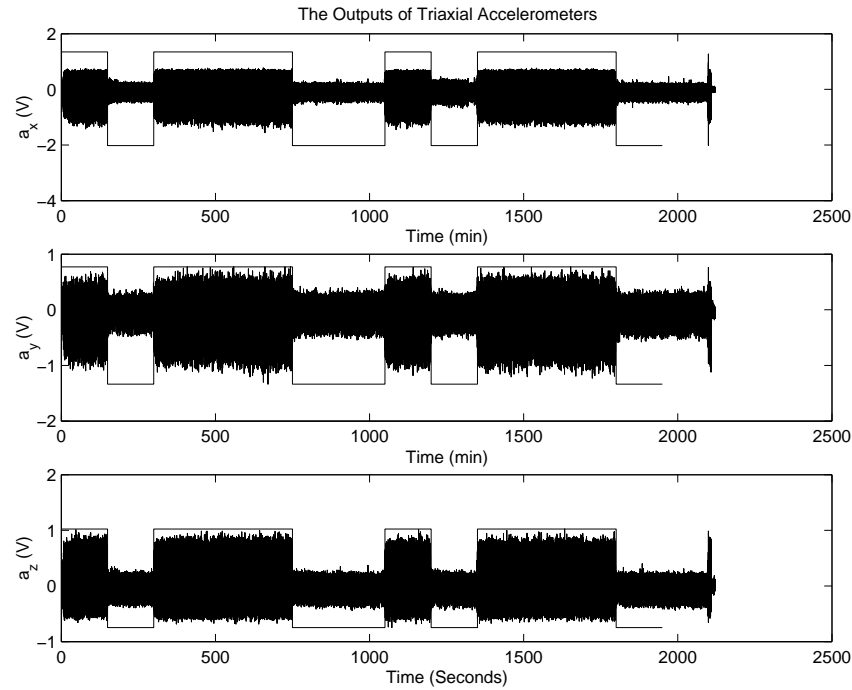

Fig. 8. The TA monitored PRBS signal 


\section{REFERENCES}

[1] J. Allin and G. Inbar. Fns parameter selection and upper limb characterization. IEEE Trans. Biomed. Eng., 33:809-817, 1986.

[2] E.W. Bai. An optimal two-stage identification algorithm for Hammerstein-Wiener nonlinear systems. Automatica, 34(3):333-338, 1998.

[3] E.W. Bai. A blind approach to the Hammerstein model identification. IEEE Tran. on Signal Processing, 50(7):1610-1619, 2002.

[4] E.W. Bai. Identification of linear systems with hard input nonlinearities of known structure. Automatica, 38:853-860, 2002.

[5] E.W. Bai. Decoupling the linear and nonlinear parts in Hammerstein model identification. Automatica, 40:671-676, 2004.

[6] L. Bernotas, P. Crago, and H. Chizeck. A discrete-time model of electrically stimulated muscle. IEEE Trans. Biomed. Eng., 33:829-838, 1986.

[7] S.A. Bilings and S.Y. Fakhouri. Identification of a class of nonlinear systems using correlation analysis. Proc. Inst. Elect. Eng., 125(7):691-697, 1978.

[8] J.J. Bussgang. Crosscorrelation functions of amplitude distorted gaussian signals. Tech. Rept. 216, MIT Res. Lab. of ElectronicsProc. IEEE EMBS, 1952.

[9] F. Chang and R. Luus. A noniterative method for identification using Hammerstein model. IEEE Trans. Automat. Contr., 16:464-468, 1971.

[10] N. Cristianini and J. Shawe-Taylor. An Introduction to Support Vector Machines and other kernel-based learning methods. Cambridge University Press, UK, 2000.

[11] K. Yanagawa D. Abe and S. Niihata. Effects of load carriage, load position, and walking speed on energy cost of walking. Applied Ergonomics, 35:329-335, 2004.

[12] E.J. Dempsey and D.T. Westwick. Identification of Hammerstein models with cubic spline nonlinearities. IEEE Trans. Biomed. Eng., 51:237-245, 2004.

[13] DI Prampero, P. Davies, P. Cerretelli, and R.Margria. An analysis of $o_{2}$ debt contracted in submaximal exercise. J. Appl. Physiol., 29:547-551, 1970.

[14] D.B. Dill. Oxygen used in horizontal and grade walking and running on the treadmill. J Appl Physiol, 20:19-22, 1965.

[15] H. Drucker, C. Burges, L.Kaufman, A. Smola, and V. Vapnik. Support vector regression machines. In M. Mozer, M. Jordan, and T. Petsche, editors, Advances in Neural Information Procession Systems, pages 155-161. Cambridge, MA, 1997.

[16] D. Efeld, U. Hoffmann, and J. Stegemann. $v o_{2}$ kinetics in subjects differing in aerobic capacity: investigation by spectral analysis. European Journal of Applied Physiology, 56(5):508-515, 1987.

[17] E. Eskinat, S.H. Johnson, and W.L. Luyben. Use of Hammerstein models in identification of nonlinear systems. AIChE J., 37:255-268, 1991.

[18] M. Espinoza, K. Pelckmans, L. Hoegaerts, J. Suykens, J. De Brabanter, and B. De Moor. A comparative study of ls-svms applied to the silver box identi.cation problem. Symposium on nonlinear control systems NOLCOS, pages 513-518, 2004.

[19] P. Falugi, L. Giarre, and G. Zappa. Approximation of the feasible parameter set in worst-case identification of Hammerstein models. Automatica, 41:1017-1024, 2005.

[20] B.A. Franklin. ACSM's Guidelines for Exercise Testing and Prescription. Lippincott Williams \& Wilkins, New York, 2000. 
[21] I. Goethals., K. Pelckmans, J.A.K. Suykens, and B. De Moor. Identification of mimo Hammerstein models using least squares support vector machines. Automatica, 41:1263-1272, 2005.

[22] Y. Guo, P.L. Bartlett, J. Shawe-Taylor, and R.C. Williamson. Covering numbers for support vector machines. IEEE Trans. on Information Theory, 48(1):239-250, 2002.

[23] J.M. Hagberg, R.C. Hickson, A.A. Ehsani, and J.0. Holloszy. Faster adjustment to and recovery from submaximal exercise in the trained state. J. Appl. Physiol., 48:218-224, 1980.

[24] A.V. Hill and H. Lupton. Muscular exercise, lactic acid and the supply and utilization of oxygen. Q. J. Med., 16:135-171, 1923.

[25] Uwe Hoffmann, Dieter Efeld, Hans-Georg Wunderlich, and Jrgen Stegemann. Dynamic linearity of $v_{2}$ responses during aerobic exercise. European Journal of Applied Physiology, 64(2):139-144, 1992.

[26] K.J. Hunt, M. Munih, N.de N. Donaldson, and F.M.D. Barr. Investigation of the Hammerstein hypothesis in the modeling of electrically stimulated muscle. IEEE Trans. Biomed. Eng., 45(8):998-1009, 1998.

[27] I.W. Hunter and M.J. Korenberg. The identification of nonlinear biological systems: Wiener and Hammerstein cascade models. Biol. Cybern., 55:135-144, 1986.

[28] M.J. Korenberg. Identification of biological cascades of linear and nonlinear systems. Proc. Midwest Symp. Circuit Theory, 18(2):1-9, 1973.

[29] M.J. Korenberg. Recent advances in the identification of nonlinear systems: minimumvariance approximation by Hammerstein models. Proc. IEEE EMBS, 13:2258-2259, 1991.

[30] G.N. Maksym, R.E. Kearney, and J.H. Bates. Nonparametric block-structured modeling of lung tissue strip mechanics. Ann Biomed Eng, 26(2):242-252, 1998.

[31] K.S. Narendra and P.G. Gallman. An iterative method for the identification of nonlinear systems using Hammerstein model. IEEE Transactions on Automatic Control, 11:546-550, 1966.

[32] B. Ninness and S. Gibson. Quantifying the accuracy of Hammerstein model estimation. Automatica, 38:2037-2051, 2002.

[33] M. Pawlak. On the series expansion approach to the identification of Hammerstein systems. IEEE Trans. Automat. Contr., 36:763-767, 1991.

[34] D.H. Pearce and H.T. Milhorn, Jr. Dynamic and steady-state respiratory responses to bicycle exercise. J. Appl. Physiol., 42(6):959-967, 1977.

[35] B. Schlkopf and A. Smola. Learning with kernels. MA: MIT Pres, Cambridge, 2002.

[36] A. Smola and B. Schlkopf. A tutorial on support vector regression. Statistics and Computing, 14:199-222, 2004.

[37] S.W. Su, J. Bao, and P.L. Lee. Control of multivariable Hammerstein systems by using feedforward passivation. Ind. \& Eng. Chem. Res., 44(4):891-899, 2005.

[38] S.W. Su, L. Wang, B. Celler, A. Savkin, and Y. Guo. Modelling and control for heart rate regulation during treadmill exercise. in Proceedings of the 28th Annual International Conference of the IEEE Engineering in Medicine and Biology Society (EMBS), pages 4299-4302, September, 2006, New York, USA.

[39] J.A.K. Suykens, Van Gestel, J. De Brabanter, B. De Moor, and J. Vandewalle. Least squares support vector machines. WorldScientific, Singapore, 2002.

[40] J.A.K. Suykens, Van Gestel, J. De Brabanter, B. De Moor, and J. Vandewalle. Least squares support vector machines. Neurocomputing, 48:85-105, 2002.

[41] W.H. T Van der Walt and C.H. Wyndham. An equation for prediction of energy expenditure of walking and running. $J$ Appl Physiol, 34:559-563, 1973. 
[42] V. Vapnik. The Nature of Statistical Learning Theory. Springer, New York, 1995.

[43] V. Vapnik. Statistical Learning Theory. John Wiley, New York, 1998.

[44] V. Vapnik and A. Lerner. Pattern recognition using generalized portrait method. Automation and Remote Control, 14, 1963.

[45] J. Voros. Iterative algorithm for parameter identification of Hammerstein systems with two-segment nonlinearities. IEEE Transactions on Automatic Control, 44:2145-2149, 1999.

[46] L. Wang, S.W. Su, B. Celler, and A. Savkin. Modeling of a gas concentration measurement system. in Proceedings of the 27th Annual International Conference of the IEEE Engineering in Medicine and Biology Society (EMBS), pages 6695-6698, September, 2005, Shanghai, China.

[47] B.J. Whipp, S.A. Ward, N. Lamarra, J.A. Davis, and K. Wasserman. Parameters of ventilatory and gas exchange dynamics during exercise. J. Appl. Physiol., 52(6):1506-1513, 1982. 